\title{
NUMERICAL MODELING OF THERMAL PROCESSES IN THE LIVING TISSUE DOMAIN SECURED WITH A LAYER OF PROTECTIVE CLOTHING
}

\author{
Bohdan Mochnacki ${ }^{1}$, Ewa Majchrzak ${ }^{2}$, Mateusz Duda $^{1}$ \\ ${ }^{1}$ Higher School of Labour Safety Management in Katowice, Katowice, Poland \\ ${ }^{2}$ Silesian University of Technology, Gliwice, Poland \\ 'bmochnacki@wszop.edu.pl
}

\begin{abstract}
In the paper the problem of thermal processes proceeding in the domain of biological tissue secured with protective clothing is discussed. In particular, the mathematical model of heat exchange corresponding to conditions of high temperature in the system environment - layer of protective clothing - air gap - skin tissue is formulated in the form of a certain boundary - initial problem. Next, the numerical algorithm based on the boundary element method is presented. In the final part of the paper the examples of numerical simulations are shown.
\end{abstract}

Keywords: bioheat transfer, protective clothing, modeling of thermal processes, boundary element method

\section{Introduction}

The clothing protecting one against the action of high (or low) temperature is often used in the various fields of human activity. For some works it is even necessary. At this point one can mention the professions of firefighter, ironmaster, caster, welder etc. In the industry practice a number of standards for the insulating efficiency of protective clothing is applied (for example the delay time to reach the high temperature to the skin surface). Tests for determining such standards concerning the different types of clothing are quite expensive and it seems that the methods of numerical simulation can be an effective tool to avoid the significant expenses. In this paper the $1 \mathrm{D}$ problem is considered (because of the geometry of domain considered this simplification is here acceptable) and the transient temperature field in the skin tissue sub-domain $T(x, t)$ is determined. On the external surface of fabric the Robin condition is assumed, while the air gap between the clothing and skin surface is taken into account by introducing the additional thermal resistance into a mathematical model. At the stage of numerical algorithm construction the I scheme of the boundary element method (BEM) is used. The examples of numerical computations are also presented. 


\section{Governing equations}

We consider the non-homogeneous domain being the composition of the skin tissue, the layer of protective clothing and the air layer between skin and fabric, while we assume that the material from which the clothing is made can be treated as the homogeneous one.

The temperature field in the tissue sub-domain is described by the following energy equation $[1,2]$ (the $1 \mathrm{D}$ problem is considered and taking into account the real geometrical conditions such simplification is quite acceptable)

$$
x \in\left(L_{0}, L\right): \quad C \frac{\partial T(x, t)}{\partial t}=\lambda \frac{\partial^{2} T(x, t)}{\partial x^{2}}+G_{B} C_{B}\left[T_{B}-T(x, t)\right]+Q_{m e t}
$$

where $C$ is the mean volumetric specific heat of the skin tissue, $\lambda$ is the mean thermal conductivity, $G_{B}$ is the perfusion coefficient, $C_{B}$ is the volumetric specific heat of blood, $Q_{\text {met }}$ is the metabolic heat source. It should be pointed out that the second component on the right-hand side of equation (1) corresponds to the so-called perfusion heat source and it is connected with the presence of the big number of capillaries in the domain of soft tissue.

The temperature distribution in the fabric sub-domain results from the well-known Fourier equation

$$
x \in\left(0, L_{0}\right): \quad C_{F} \frac{\partial T_{F}(x, t)}{\partial t}=\lambda_{F} \frac{\partial^{2} T_{F}(x, t)}{\partial x^{2}}
$$

where $C_{F}$ is the volumetric specific heat of the fabric, $\lambda_{F}$ is the thermal conductivity. On the external surface of the fabric the Robin condition can be assumed

$$
x=0: \quad q_{F 0}(x, t)=\alpha_{s}\left[T_{F}(x, t)-T_{a}\right]
$$

where $\alpha_{s}$ is the substitute heat transfer coefficient, while $T_{a}$ is the ambient temperature. For $x=L_{0}$ the thermal resistance of the air gap is defined as the inverse of the heat transfer coefficient $\alpha_{\text {gap }}$ (see: Section 3), this means $R=1 / \alpha_{\text {gap }}$ and the boundary condition at this point has a form

$$
x=L_{0}: \quad q_{F}(x, t)=\frac{T_{F}(x, t)-T(x, t)}{R}=q(x, t)
$$

or

$$
x=L_{0}: \quad\left\{\begin{array}{c}
q_{F}(x, t)=q(x, t) \\
T(x, t)=T_{F}(x, t)-R q(x, t)
\end{array}\right.
$$


At the point corresponding to the right edge of domain one has

$$
x=L: \quad T(x, t)=T_{\text {in }}
$$

where $T_{i n}$ is the temperature of body interior.

The mathematical model should be supplemented by the initial conditions. In domain of fabric and tissue the initial temperatures are given, in particular

$$
t=0: \quad T_{F}(x, 0)=T_{F 0}
$$

The initial condition for the tissue is the following

$$
t=0: \quad T(x, 0)=T_{0}(x)
$$

and the function $T_{0}(x)$ is assumed in the form of quadratic function [3].

\section{Boundary element method}

In the first place the BEM algorithm for the skin tissue sub-domain will be presented. So, the equation (1) can be written in the form

$$
L_{0}<x<L: \quad C \frac{\partial T(x, t)}{\partial t}=\lambda \frac{\partial^{2} T(x, t)}{\partial x^{2}}+Q(x, t)
$$

where $Q(x, t)=G_{B} C_{B}\left[T_{B}-T(x, t)\right]+Q_{m e t}$ is the source term.

Let us introduce the time grid with the constant step $\Delta t=t^{f}-t^{f-1}$. Using the first scheme of the BEM for the transition $t^{f-1} \rightarrow t^{f}, f=1,2, \ldots, F$, one obtains the following boundary integral equation $[4,5]$

$$
\begin{gathered}
T\left(\xi, t^{f}\right)+\left[\frac{1}{C} \int_{t^{f-1}}^{t^{f}} T^{*}\left(\xi, x, t^{f}, t\right) q(x, t) \mathrm{d} t\right]_{x=L_{0}}^{x=L}= \\
{\left[\frac{1}{C} \int_{t^{f-1}}^{t^{f}} q^{*}\left(\xi, x, t^{f}, t\right) T(x, t) \mathrm{d} t\right]_{x=L_{0}}^{x=L}+\int_{L_{0}}^{L} T^{*}\left(\xi, x, t^{f}, t^{f-1}\right) T\left(x, t^{f-1}\right) \mathrm{d} x+} \\
\frac{1}{C} \int_{t^{f-1}}^{t^{f}} \int_{L_{0}}^{L} Q(x, t) T^{*}\left(\xi, x, t^{f}, t\right) \mathrm{d} x \mathrm{~d} t
\end{gathered}
$$

where $\xi \in\left(L_{0}, L\right)$ is the observation point, $T^{*}\left(\xi, x, t^{f}, t\right)$ is the fundamental solution, $q^{*}\left(\xi, x, t^{f}, t\right)=-\lambda \partial T^{*}\left(\xi, x, t^{f}, t\right) / \partial x$ and $q\left(x, t^{f}\right)=-\lambda \partial T\left(x, t^{f}\right) / \partial x$. 
For the task considered the fundamental solution has the form

$$
T^{*}\left(\xi, x, t^{f}, t\right)=\frac{1}{2 \sqrt{\pi a \Delta t}} \exp \left[-\frac{(x-\xi)^{2}}{4 a \Delta t}\right]
$$

where $a=\lambda / C$ is the heat diffusion coefficient.

The heat flux $q^{*}\left(\xi, x, t^{f}, t\right)$ resulting from the fundamental solution can be calculated analytically [4]

$$
q^{*}\left(\xi, x, t^{f}, t\right)=\frac{\lambda(x-\xi)}{4 \sqrt{\pi}(a \Delta t)^{3 / 2}} \exp \left[-\frac{(x-\xi)^{2}}{4 a \Delta t}\right]
$$

Using the constant elements (with respect to time) one obtains

$$
\begin{gathered}
T\left(\xi, t^{f}\right)+\left[q\left(x, t^{f}\right) g(\xi, x)\right]_{x=L_{0}}^{x=L}=\left[T\left(x, t^{f}\right) h(\xi, x)\right]_{x=L_{0}}^{x=L}+ \\
\int_{L_{0}}^{L} T^{*}\left(\xi, x, t^{f}, t^{f-1}\right) T\left(x, t^{f-1}\right) \mathrm{d} x+\int_{L_{0}}^{L} Q\left(x, t^{f-1}\right) g(\xi, x) \mathrm{d} x
\end{gathered}
$$

where

$$
g(\xi, x)=\frac{1}{C} \int_{t^{f-1}}^{t^{f}} T^{*}\left(\xi, x, t^{f}, t\right) \mathrm{d} t, \quad h(\xi, x)=\frac{1}{C} \int_{t^{f-1}}^{t^{f}} q^{*}\left(\xi, x, t^{f}, t\right) \mathrm{d} t
$$

Additionally, we denote

$$
P(\xi)=\int_{L_{0}}^{L} T^{*}\left(\xi, x, t^{f}, t^{f-1}\right) T\left(x, t^{f-1}\right) \mathrm{d} x, \quad Z(\xi)=\int_{L_{0}}^{L} Q\left(x, t^{f-1}\right) g(\xi, x) \mathrm{d} x
$$

For $\xi \rightarrow L^{-}$and $\xi \rightarrow L_{0}^{+}$one obtains the equations

$$
\begin{gathered}
{\left[\begin{array}{cc}
-g_{s}\left(L_{0}, L_{0}\right) & g_{s}\left(L_{0}, L\right) \\
-g_{s}\left(L, L_{0}\right) & g_{s}(L, L)
\end{array}\right]\left[\begin{array}{c}
q\left(L_{0}, t^{f}\right) \\
q\left(L, t^{f}\right)
\end{array}\right]=} \\
{\left[\begin{array}{cc}
-h\left(L_{0}^{+}, L_{0}\right)-1 & h\left(L_{0}^{+}, L\right) \\
-h\left(L^{-}, L_{0}\right) & h\left(L^{-}, L\right)-1
\end{array}\right]\left[\begin{array}{c}
T\left(L_{0}, t^{f}\right) \\
T\left(L, t^{f}\right)
\end{array}\right]+} \\
{\left[\begin{array}{c}
P\left(L_{0}\right) \\
P(L)
\end{array}\right]+\left[\begin{array}{c}
Z\left(L_{0}\right) \\
Z(L)
\end{array}\right]}
\end{gathered}
$$


or

$$
\left[\begin{array}{ll}
G_{11} & G_{12} \\
G_{21} & G_{22}
\end{array}\right]\left[\begin{array}{c}
q\left(L_{0}, t^{f}\right) \\
q\left(L, t^{f}\right)
\end{array}\right]=\left[\begin{array}{ll}
H_{11} & H_{12} \\
H_{21} & H_{22}
\end{array}\right]\left[\begin{array}{c}
T\left(L_{0}, t^{f}\right) \\
T\left(L, t^{f}\right)
\end{array}\right]+\left[\begin{array}{c}
P\left(L_{0}\right) \\
P(L)
\end{array}\right]+\left[\begin{array}{c}
Z_{s}\left(L_{0}\right) \\
Z_{s}(L)
\end{array}\right]
$$

The system (17) allows one to find the 'missing' boundary values $T\left(x, t^{f}\right)$ or $q\left(x, t^{f}\right)$ for $x=L_{0}$ and $x=L$.

The last stage of the BEM algorithm relies on the computations of $T\left(x, t^{f}\right)$ at the optional set of internal points $\xi \in\left(L_{0}, L\right)$ using the equation [4]

$$
\begin{gathered}
T\left(\xi, t^{f}\right)=h(\xi, L) T\left(L, t^{f}\right)-h\left(\xi, L_{0}\right) T\left(L_{0}, t^{f}\right)- \\
g(\xi, L) q\left(L, t^{f}\right)+g\left(\xi, L_{0}\right) q\left(L_{0}, t^{f}\right)+P(\xi)+Z(\xi)
\end{gathered}
$$

The boundary integral equation describing the temperature field in the fabric sub-domain is simpler (because the source term is equal to zero). So

$$
\begin{gathered}
T_{F}\left(\xi, t^{f}\right)+\left[\frac{1}{C_{F}} \int_{t^{f-1}}^{t^{f}} T_{F}^{*}\left(\xi, x, t^{f}, t\right) q_{F}(x, t) \mathrm{d} t\right]_{x=0}^{x=L_{0}}= \\
{\left[\frac{1}{C_{F}} \int_{t^{f-1}}^{t^{f}} q_{F}^{*}\left(\xi, x, t^{f}, t\right) T_{F}(x, t) \mathrm{d} t\right]_{x=0}^{x=L_{0}}+\int_{0}^{L_{0}} T_{F}^{*}\left(\xi, x, t^{f}, t^{f-1}\right) T_{F}\left(x, t^{f-1}\right) \mathrm{d} x}
\end{gathered}
$$

Proceeding as before one obtains the system of equations

$$
\left[\begin{array}{ll}
G_{11}^{F} & G_{12}^{F} \\
G_{21}^{F} & G_{22}^{F}
\end{array}\right]\left[\begin{array}{c}
q_{F}\left(0, t^{f}\right) \\
q_{F}\left(L_{0}, t^{f}\right)
\end{array}\right]=\left[\begin{array}{cc}
H_{11}^{F} & H_{12}^{F} \\
H_{21}^{F} & H_{22}^{F}
\end{array}\right]\left[\begin{array}{c}
T_{F}\left(0, t^{f}\right) \\
T_{F}\left(L_{0}, t^{f}\right)
\end{array}\right]+\left[\begin{array}{c}
P_{F}(0) \\
P_{F}\left(L_{0}\right)
\end{array}\right]
$$

Now the boundary conditions should be introduced. The condition on the contact surface between fabric and skin tissue constitutes the element coupling the equations (17) and (20). Thus

$$
\left[\begin{array}{cccc}
\alpha G_{11}^{F}-H_{11}^{F} & -H_{12}^{F} & G_{12}^{F} & 0 \\
\alpha G_{21}^{F}-H_{21}^{F} & -H_{22}^{F} & G_{22}^{F} & 0 \\
0 & -H_{11} & G_{11}+R H_{11} & G_{12} \\
0 & -H_{21}^{s} & G_{21}+R H_{21} & G_{22}
\end{array}\right]\left[\begin{array}{c}
T_{F}\left(0, t^{f}\right) \\
T_{F}\left(L_{0}, t^{f}\right) \\
q\left(L_{0}, t^{f}\right) \\
q\left(L, t^{f}\right)
\end{array}\right]=\left[\begin{array}{c}
\alpha G_{11}^{F} T_{a}+P_{F}(0) \\
\alpha G_{21}^{F} T_{a}+P_{F}\left(L_{0}\right) \\
H_{12} T_{i n}+P\left(L_{0}\right)+Z\left(L_{0}\right) \\
H_{22} T_{i n}+P(L)+Z(L)
\end{array}\right]
$$


After the dissolution of the system (21) one can determine the temperature $T\left(L_{0}, t^{f}\right)$ using the formula

$$
T\left(L_{0}, t^{f}\right)=T_{F}\left(L_{0}, t^{f}\right)-R q\left(L_{0}, t^{f}\right)
$$

The heat flux $q_{F}\left(0, t^{f}\right)$ can be also found

$$
q_{F 0}\left(0, t^{f}\right)=\alpha_{s}\left[T_{F}\left(0, t^{f}\right)-T_{a}\right]
$$

The last stage of the algorithm proposed relies on the computations of internal temperatures separately for the fabric and tissue.

\section{Example of computations}

At first the problem of heat transfer coefficients appearing in the conditions (3) and (4) will be explained. The boundary heat flux is a sum of two components, namely

$$
x=0: \quad q_{c}(0, t)=\alpha_{c}\left[T_{F}(0, t)-T_{a}\right]
$$

where $q_{c}(0, t)$ is the convective component ( $\alpha_{c}$ is the heat transfer coefficient, $T_{a}$ is the ambient temperature). The second component of the boundary heat flux results from the radiation phenomenon

$$
x=0: \quad q_{r}(0, t)=\sigma \varepsilon_{g}\left[T_{F}^{4}(0, t)-T_{g}^{4}\right]-\sigma \varepsilon_{F} \varphi_{F-a}\left(1-\varepsilon_{g}\right)\left[T_{F}^{4}(0, t)-T_{a}^{4}\right]
$$

where $\sigma$ is the Stefan-Boltzmann constant, $\varepsilon_{g}, \varepsilon_{F}$ denote the emissivity of the hot gases surrounding the fabric and the emissivity of the fabric, $T_{g}, T_{a}$ are the temperatures of the hot gases and air, $\varphi_{F-g}$ is the view factor. In literature [6] one can find the simplified formula determining the boundary heat flux

$$
x=0: \quad q_{F 0}(0, t)=q_{c}(0, t)+q_{r}(0, t)=\alpha_{s}\left[T_{F}(0, t)-T_{a}\right]
$$

where $\alpha_{s}$ is the substitute heat transfer coefficient which can be found experimentally [6].

Between the fabric and skin surface is the 'trapped' air layer. The boundary condition at this region can be assumed in the form of the heat flux continuity condition. The thermal resistance of air gap resistance appearing in the condition (4) is equal to $1 / \alpha_{\text {gap }}$. At the stage of numerical computations it is assumed that 
the thermal resistance is a constant value $R=0.077 \mathrm{~m}^{2} \mathrm{~K} / \mathrm{W}$ (the authors have developed the procedure of the exact computation of temperature-dependent thermal resistance $R$, but the assumption of the constant value proposed is quite acceptable) In this paper the remaining input data are the following: thickness of tissue domain $0.012 \mathrm{~m}$, thickness of fabric $0.00677 \mathrm{~m}$, volumetric specific heat of tissue $C=3 \mathrm{MJ} /\left(\mathrm{m}^{3} \mathrm{~K}\right)$, thermal conductivity of tissue $\lambda=0.3 \mathrm{~W} /(\mathrm{mK})$ volumetric specific heat of fabric $C_{F}=0.24 \mathrm{MJ} /\left(\mathrm{m}^{3} \mathrm{~K}\right)$ thermal conductivity of fabric $\lambda_{F}=0.17 \mathrm{~W} /(\mathrm{mK})$, volumetric specific heat of blood $C_{B}=4 \mathrm{MJ} /\left(\mathrm{m}^{3} \mathrm{~K}\right)$, perfusion coefficient $G_{B}=0.00051 / \mathrm{s}$, the metabolic heat source $Q_{m e t}=245 \mathrm{~W} / \mathrm{m}^{3}$, the tissue temperature for $x=L: T_{i n}=37^{\circ} \mathrm{C}$, the heat transfer coefficient on the external surface of the fabric $\alpha_{s}=100 \mathrm{~W} /\left(\mathrm{m}^{2} \mathrm{~K}\right)$. In Figure 1 the example of the results obtained is presented. In particular the heating curves at the points corresponding to the skin surface and the internal surface of fabric (for the ambient temperature $T_{a}=80^{\circ} \mathrm{C}$ ) are shown.

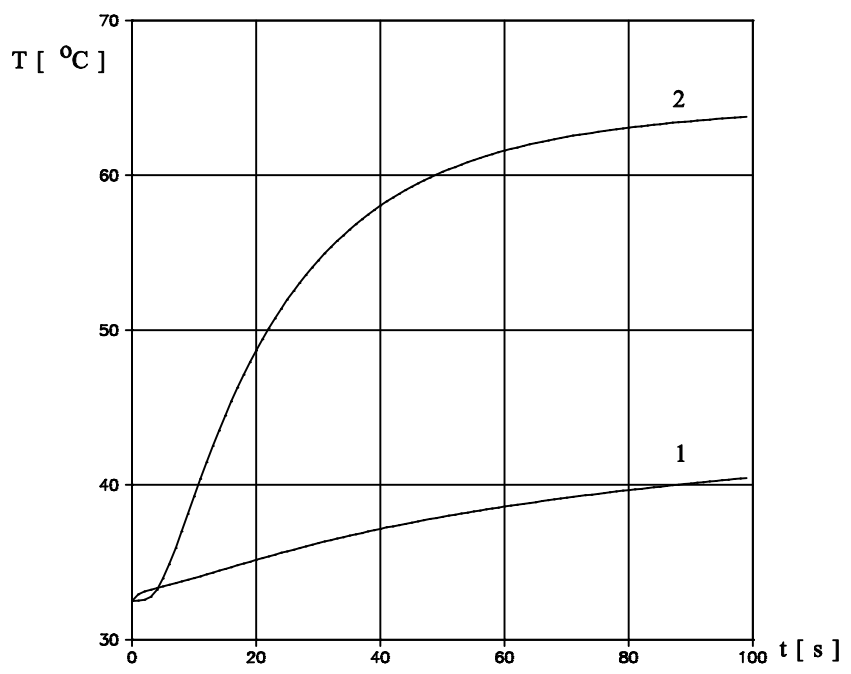

Fig. 1. Heating curves: 1 - skin, 2 - fabric

\section{Final remarks}

The algorithm presented can be used for numerical modeling of thermal processes proceeding in the domain of skin tissue protected by clothing. The different conditions can be considered and it is also possible to take into account the heterogeneity of both the fabric (the layers) and the skin tissue. The essential problem which requires the additional research is connected with the proper choice of the heat transfer coefficient on the external surface of the clothing. 


\section{Acknowledgements}

The paper is a part of Research Project PB3/2013 sponsored by WSZOP Katowice.

\section{References}

[1] Pennes H.H., Analysis of tissue and arterial blood temperatures in the resting human forearm, Journal of Applied Physiology 1948, 1, 93-122.

[2] Majchrzak E., Application of different variants of the BEM in numerical modeling of bioheat transfer processes, MCB: Molecular \& Cellular Biomechanics 2013, 10, 3, 201-232.

[3] Majchrzak E., Mochnacki B., Jasiński M., Numerical modelling of bioheat transfer in multi-layer skin tissue domain subjected to a flash fire, Computational Fluid and Solid Mechanics, Vol. II, Elsevier, 2003, 1766-1770.

[4] Majchrzak E., Metoda elementów brzegowych w przepływie ciepła, Wydawnictwo Politechniki Częstochowskiej, Częstochowa 2001.

[5] Majchrzak E., Dziewoński M., Kałuża G., Application of the boundary element method coupled with the artificial source procedure for numerical modelling of freezing process, Scientific Research of the Institute of Mathematics and Computer Science, Czestochowa University of Technology 2009, 1(8), 105-112.

[6] Chitphiromsri P., Modeling of thermal performance firefighter protecting clothing during the intense heat exposure, Mechanical Engineering, Doctoral Theses, Raleigh 2004. 